\title{
Knockdown of SUMO-activating enzyme subunit 2 (SAE2) suppresses cancer malignancy and enhances chemotherapy sensitivity in small cell lung cancer
}

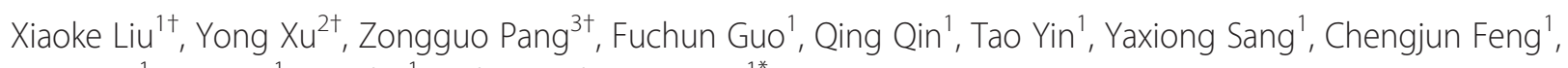
Xiaoyu Li', Li Jiang ${ }^{1}$, Pei Shu' ${ }^{1}$ and Yongsheng Wang ${ }^{1 *}$

\begin{abstract}
Background: SUMO-activating enzyme subunit 2 (SAE2) is the sole E1-activating enzyme required for numerous important protein SUMOylation, abnormal of which is associated with carcinogenesis. SAE2 inactivation was recently reported to be a therapeutic strategy in cancers with Myc overexpression. However, the roles of SAE2 in small cell lung cancer (SCLC) are largely unknown.

Methods: Stably SAE2 knockdown in H446 cells were established with a lentiviral system. Cell viability, cell cycle, and apoptosis were analyzed using MTT assay and flow cytometric assay. Expression of SAE2 mRNA and protein were detected by $\mathrm{QPCR}$, western blotting, and immunohistochemical staining. Cell invasion and migration assay were determined by transwell chamber assay. $\mathrm{H} 446$ cells with or without SAE2 knockdown, nude mice models were established to observe tumorigenesis.

Results: SAE2 was highly expressed in SCLC and significantly correlated with tumorigenesis in vivo. Cancer cells with RNAi-mediated reduction of SAE2 expression exhibited growth retardation and apoptosis increasing. Furthermore, down-regulation of SAE2 expression inhibited migration and invasion, simultaneously increased the sensitivity of H446 to etoposide and cisplatin.
\end{abstract}

Conclusions: SAE2 plays an important role in tumor growth, metastasis, and chemotherapy sensitivity of $\mathrm{H} 446$ and is a potential clinical biomarker and therapeutic target in SCLC with high c-Myc expression.

Keywords: SUMO-activating enzyme subunit 2, Small cell lung cancer (SCLC), Chemotherapy sensitivity

\section{Background}

Lung cancer is the first leading cause of cancer-related deaths in males while second in females all over the world $[1,2]$. Small cell lung cancer (SCLC) accounts for $13 \%$ of all newly diagnosed cases of lung cancer worldwide, representing approximately 180,000 cases per year [3-5]. Patients at extensive stage have median survival of 7-12 months, and 5-year survival is only 1-2\%. Whereas among patients at limited stage, median survival is about

\footnotetext{
*Correspondence: wangys@scu.edu.cn

${ }^{\dagger}$ Equal contributors

'Department of Thoracic Oncology, Cancer Center, State Key Laboratory of Biotherapy/Collaborative Innovation Center of Biotherapy, West China Hospital, Sichuan University, Chengdu, Sichuan, People's Republic of China Full list of author information is available at the end of the article
}

23 months and 5-year survival is $12-25 \%$ [6-10]. SCLC is the most aggressive type of lung cancer mainly due to rapid growth, wide invasion, and fast metastasis [8, 11-13]. Therefore, it is critical to investigate an effective strategy for SCLC treatment.

It is widely reported that SUMOylation is a posttranslational modification, which is significantly involved in diverse cellular functions, including genome integrity, nuclear transport, gene expression, signal transduction, and cell proliferation and differentiation through modulating protein-protein interactions [14-19]. In addition, recent data have pointed that cancer is associated with alterations in SUMOylation [14]. Mechanically, SUMOylation requires three steps of enzymatic reactions to

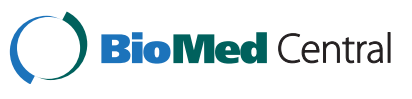


attach the small ubiquitin modifier (SUMO) protein to the substrates: activation with the E1 enzyme (SAE1/ SAE2), conjugation with the E2 enzyme (UBC9), and ligation with E3 ligase. Especially, SAE2 is a critical component of the SUMO-activating enzyme which is necessary for SUMO pathway [15, 18-20]. Accumulating evidence indicates that SUMO pathway is involved in a variety of cancers [21-30]. A recent study showed that SAE2 inactivation could be a therapeutic strategy in Myc overexpression cancers [31]. However, the roles of SAE2 in SCLC, in which c-Myc was widely amplified and over-expressed [32-40], are still unknown.

Here, we investigated the role of SAE2 in SCLC. We found higher expression of SAE2 in SCLC than in normal tissues. Furthermore, we observed that down-regulation of SAE2 expression in SCLC cells suppressed cell proliferation, migration, invasion as well as tumor formation and promoted cell apoptosis. Based on these findings, we concluded that down-regulation of SAE2 expression enhanced tumor suppression and sensitivity of chemotherapy in SCLC, and targeting SAE2 may be a new method for patients with SCLC.

\section{Results}

Increased expression of SAE2 in SCLC patients and cell lines

To investigate the roles of SAE2 in SCLC in which cMyc was demonstrated to be widely amplified and expressed, we detected SAE2 protein level by immunohistochemical staining in the SCLC specimens and the normal lung tissues. Interestingly, we detected a significant elevated expression of SAE2 in SCLC tumor tis$\operatorname{sues}(P<0.001)$ (Fig. 1a). Moreover, we analyzed gene expression of SAE2 from the NCBI GEO database with 23 clinical small cell lung cancer (SCLC) samples from patients undergoing pulmonary resection and 42 normal tissue samples including the lung using Affymetrix $\mathrm{Hu}-$ man Genome U133 Plus 2.0 Array (GSE43346). SAE2 was also highly expressed in SCLC compared to the normal tissues (Additional file 1: Figure S1). The mRNA and protein level of SAE2 were detected using quantitative real-time PCR and Western blot in several cell lines, including H446, H526, H69, H146, and BEAS-2B. Both mRNA expression and protein levels of SAE2 were significantly higher in SCLC cell lines compared with normal cell line (BEAS-2B) (Fig. 1b, c).These results indicated that SAE2 is highly expressed in SCLC tissues and cell lines.

\section{Inhibition of cell proliferation in H446 cells with SAE2 silence}

To investigate the role of SAE2 in SCLC, we firstly established H446 cells with stably down-expressing SAE2 (shSAE2-H446) by Plko.1-shSAE2. Cells stably harbored the corresponding empty Plko.1 vector which was established as control (shCtrl-H446). Quantitative real-time PCR and Western blotting analysis showed that the

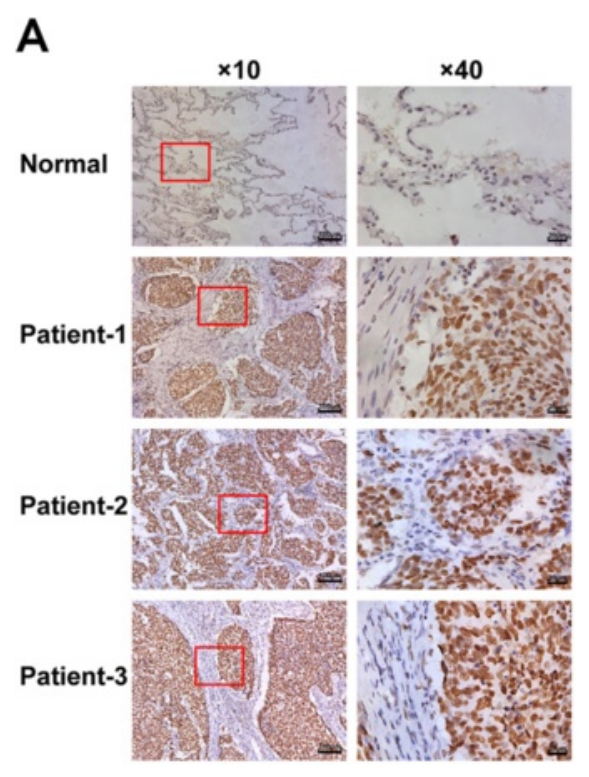

B
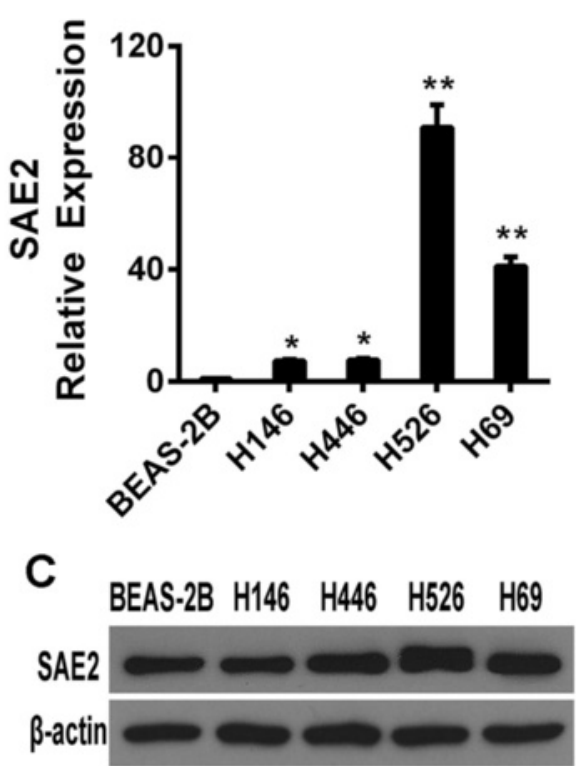

Fig. 1 SAE2 expression in SCLC tissues and cell lines. a Representative immunohistochemical results of the expression of SAE2 in tumor tissues from SCLC patient $(n=20)$ and normal lung tissues $(n=5)$. b The expression of SAE2 mRNA in SCLC cell lines (H446, H146, H526 H69, and BEAS-2B). c The expression of SAE2 protein in SCLC cell lines (H446, H146, H526, H69, and BEAS-2B). Data represent means \pm SEM of three independent experiments $\left({ }^{*} P<0.05,{ }^{* *} P<0.01\right)$ 
expression of SAE2 was markedly decreased in shSAE2H446 cells (Fig. 2a, b). We further examined the effect of SAE2 on cell proliferation determined by the MTT assay. The growth rate revealed that silence of SAE2 significantly reduced viable cells (Fig. 2c). Consistently, less numbers of colonies were observed in shSAE2-H446 cells in colony formation assay (Fig. 2d), and the difference was significant (Fig. 2e).These results suggest that silence of SAE2 inhibits the growth of SCLC cell.

\section{Induction of apoptosis in H446 with SAE2 knockdown}

To explore the effect of SAE2 deficiency on cell apoptosis and cell cycle, apoptosis assay by Annexin VFITC/propidium iodide (PI) staining and propidium iodide (PI) staining were performed. Our results revealed that there were approximately $20 \%$ apoptotic cells in shSAE2-H446 cells (Fig. 3a, second panel), compared to only $9.39 \%$ of cells in shCtrl-H446 cells (Fig. 3a, first panel). Meanwhile, we detected proteins involved in apoptosis by Western blot. Expression of Bcl-2 was prominently decreased, while Bcl-XL, P53, and P21 were maintained (Fig. 3c). These data indicated that silence of SAE2 was sufficient to promote apoptosis by decreasing the expression of Bcl-2 in H446 cells. In addition, there was no significant difference in cell cycle of shSAE2H446 cells compared with shCtrl-H446 cells after starving for $24 \mathrm{~h}$, detected by PI staining (Fig. 3d, e). We conclude that knockdown of SAE2 in SCLC cells increased apoptosis.

\section{Knockdown of SAE2-inhibited cell invasion and migration in vitro and tumorigenesis in vivo}

We next investigated the effects of SAE2 on cell invasion and migration. A transwell cell migration assay showed that knockdown of SAE2 in H446 cells exhibited a significant decrease in cell migration ability (Fig. 4a). Furthermore, by using a transwell matrigel cell invasion assay, we found that the invasion ability of shSAE2H446 cells was also significantly reduced (Fig. 4a, b). As MMP2 and MMP9 were crucial proteins involved in cancer cell metastasis, we reasoned that SAE2 might regulate MMP expression in the SCLC. Expression of MMP2 and MMP9 in shCtrl-H446 cells or shSAE2H446 cells were measured by Western blot analysis. We

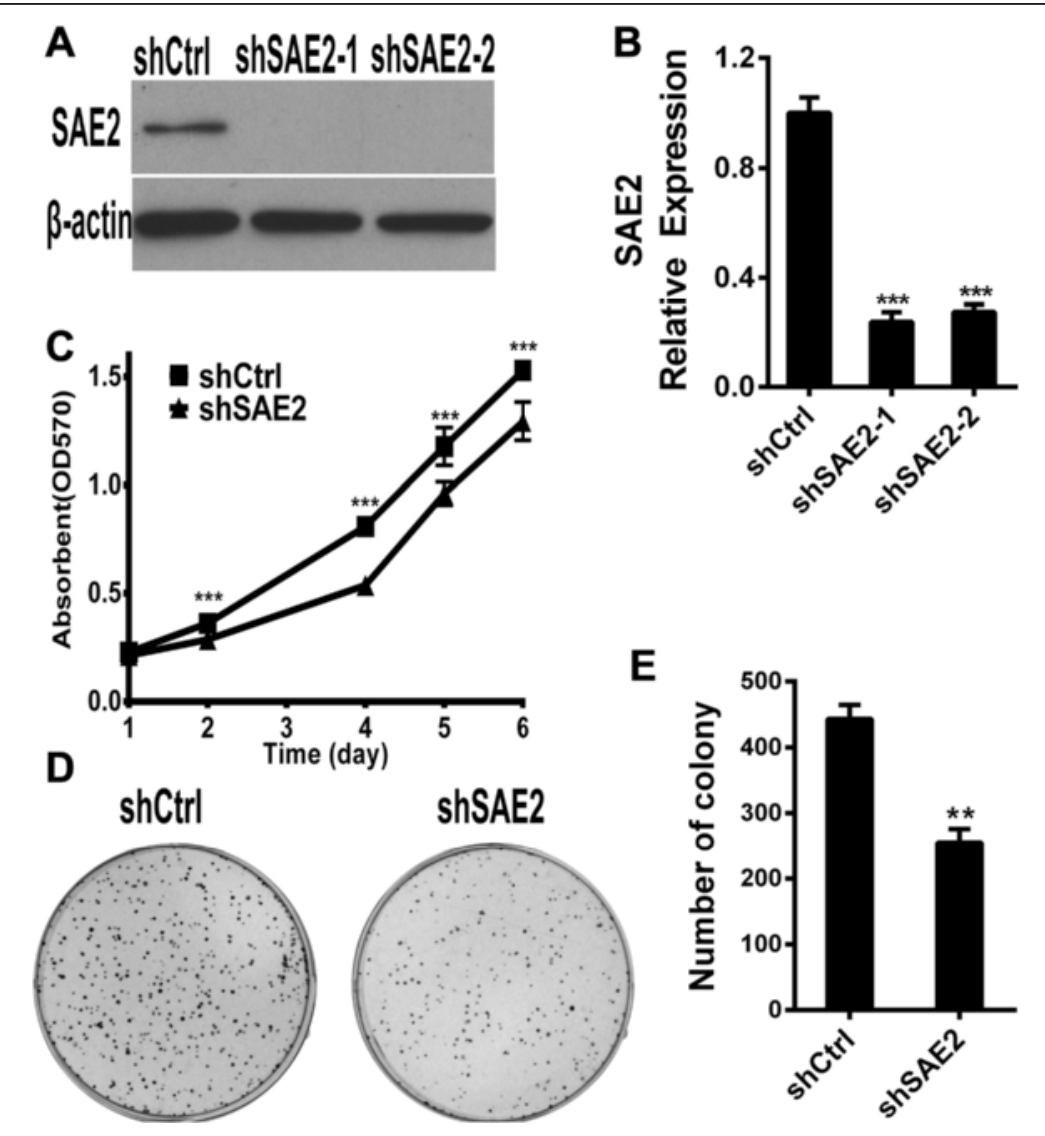

Fig. 2 SAE2 affects the proliferation of SCLC cell line. Knockdown of SAE2 in H446 cell line confirmed by Western blot (a) and real-time PCR (b). c Growth rate of H446 cells with or without knockdown of SAE2 was determined by MTT assay. Data shown are means \pm SD of three independent experiments. Representative colony images (d) and quantification of colony (e) are shown with or without knockdown of SAE2. Data are presented as means \pm SD of three independent experiments $\left({ }^{* *} P<0.01\right.$, $\left.{ }^{* * *} P<0.001\right)$ 

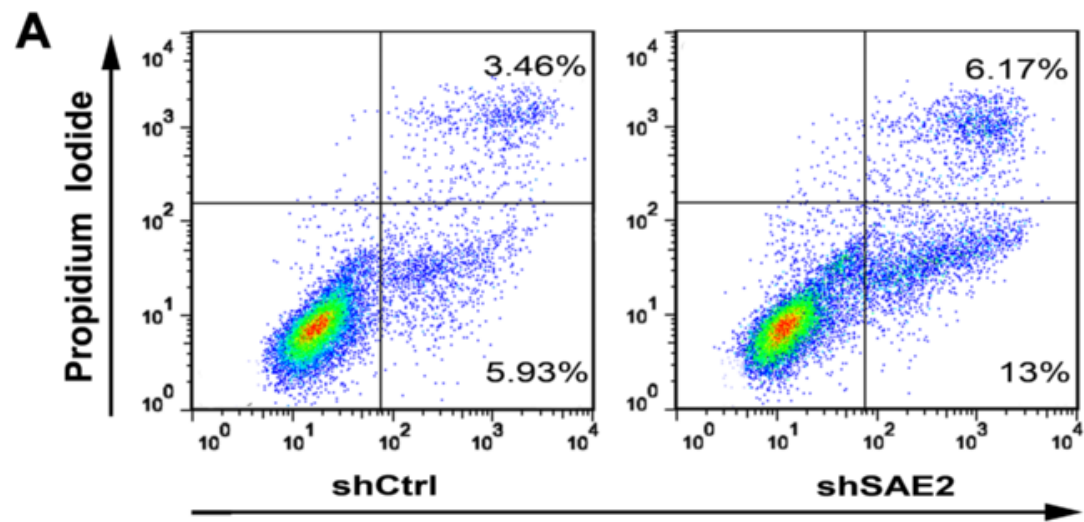

B

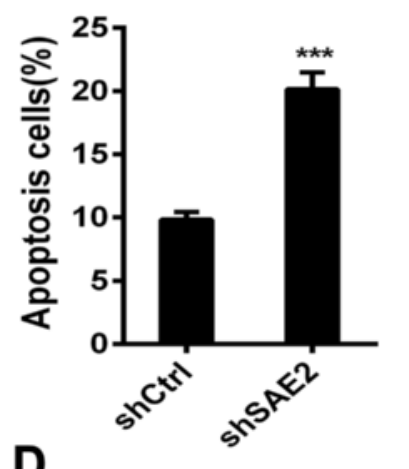

D

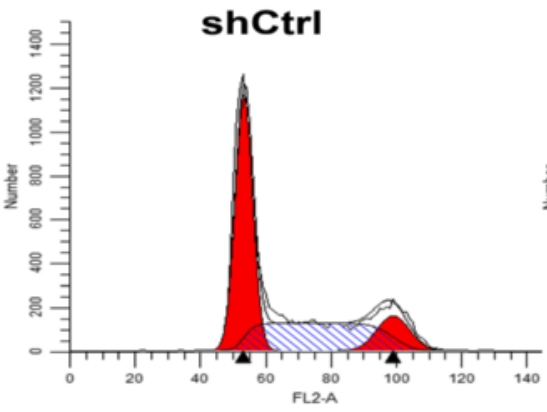

C

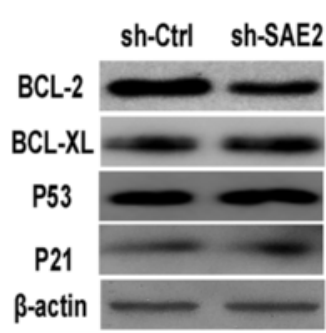

E
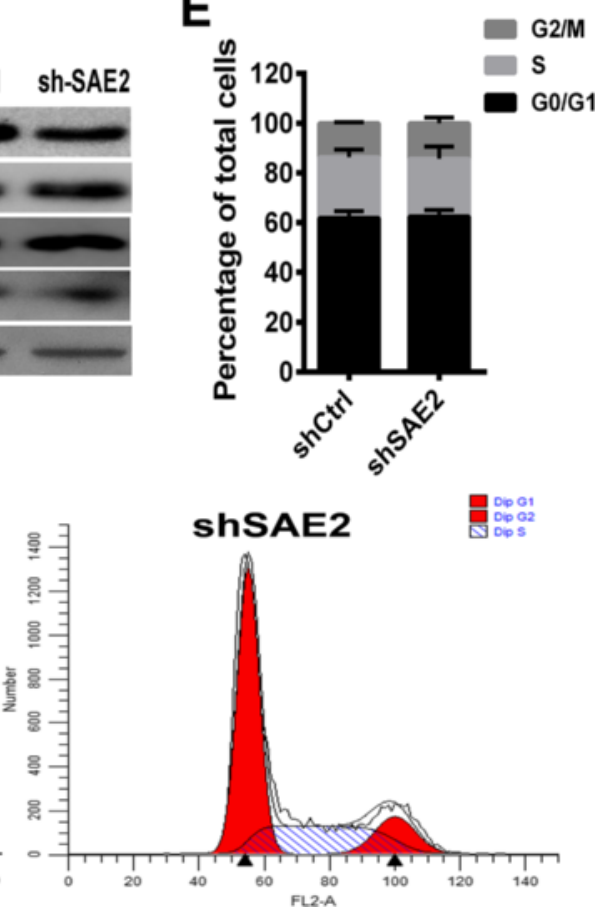

Fig. 3 SAE2 is associated with apoptosis in SCLC cell line. a Representative FCM result stained by Annexin V-FITC and PI. Annexin $\mathrm{V}^{+} / \mathrm{PI}{ }^{-}$and Annexin $\mathrm{V}^{+} / \mathrm{PI}^{+}$cells were designed as early stage and advanced stage of the apoptotic process. $\mathbf{b}$ The flow cytometry (FCM) results are presented as the percentage of apoptotic cells. The sum of FITC-positive cells in the top right and bottom right quadrants represents the total percentage of apoptotic cells. c Apoptosis-related protein levels were examined by Western blots using $\beta$-actin as a loading control. d Cell cycle analysis was performed by FCM and $\mathbf{e}$ the percentage of the cell population at different cell cycle phases was shown. Each data point represents means \pm SD of three independent experiments $\left({ }^{* *} P<0.001\right)$

found that the levels of MMP2 and MMP9 were decreased in shSAE2-H446 cells compared with that in shCtrl-H446 cells (Fig. 4c). These data indicated that silence of SAE2 was sufficient to inhibit invasion and migration by decreasing the expression of MMP2 and MMP9 in H446 cells. Furthermore, to test the effects of knockdown of SAE2 in vivo, shCtrl-H446 cells or shSAE2-H446 cells were inoculated subcutaneously into the flanks of nude mice, and the tumorigenesis in mice was observed for 8 weeks (Fig. 5). As the results shown in Table 1, the incidence of subcutaneous tumorigenesis in the athymic nude mice harboring shSAE2-H446 cells was $0 / 15$ at 56 days post-inoculation, whereas $14 / 15$ xenograft were established with shCtrl-H446 cells. These results demonstrated that knockdown of SAE2 markedly inhibited tumorigenesis of $\mathrm{H} 446$ cells in vivo.

Sensitization of chemotherapy in H446 cell line with SAE2 knockdown

It is widely reported that SCLC is the most aggressive type of lung cancer mainly due to quickly refractory to initial therapy. We next investigated whether knockdown 
A
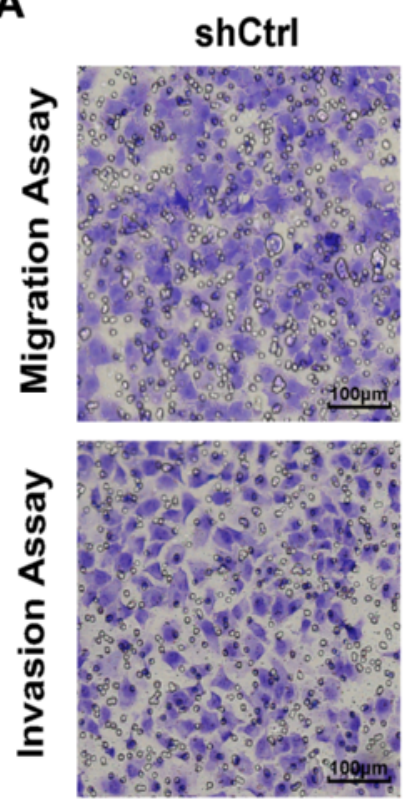

ShSAE2
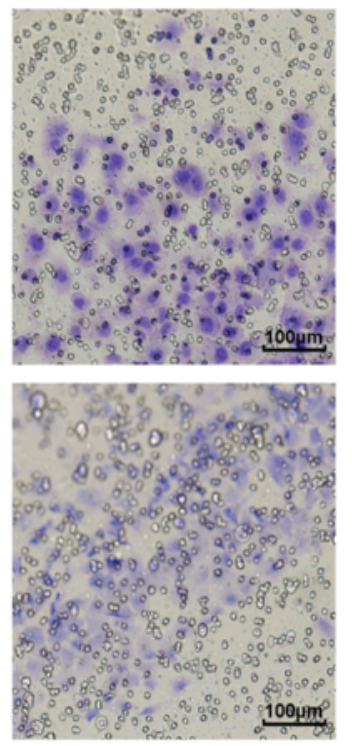

B

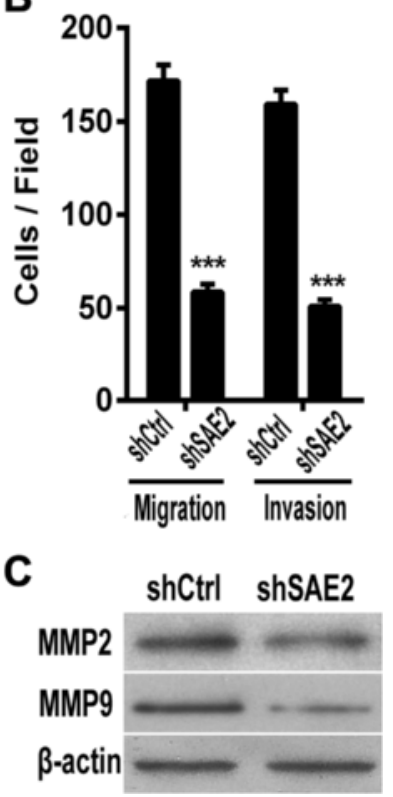

Fig. 4 SAE2 is essential for the migratory and invasive potential of SCLC cells in vitro. a Transwell invasion and migration assays of shCtrl-H446 and shSEA2 H446 tumor cells were performed. $\mathbf{b}$ The results are showed as an average of the number of migration/invasion cells from six random microscopic fields. c MMP2 and MMP9 protein level was measured by Western blot. Each data point represents mean \pm SD of three independent experiments $\left({ }^{* * *} P<0.001\right)$

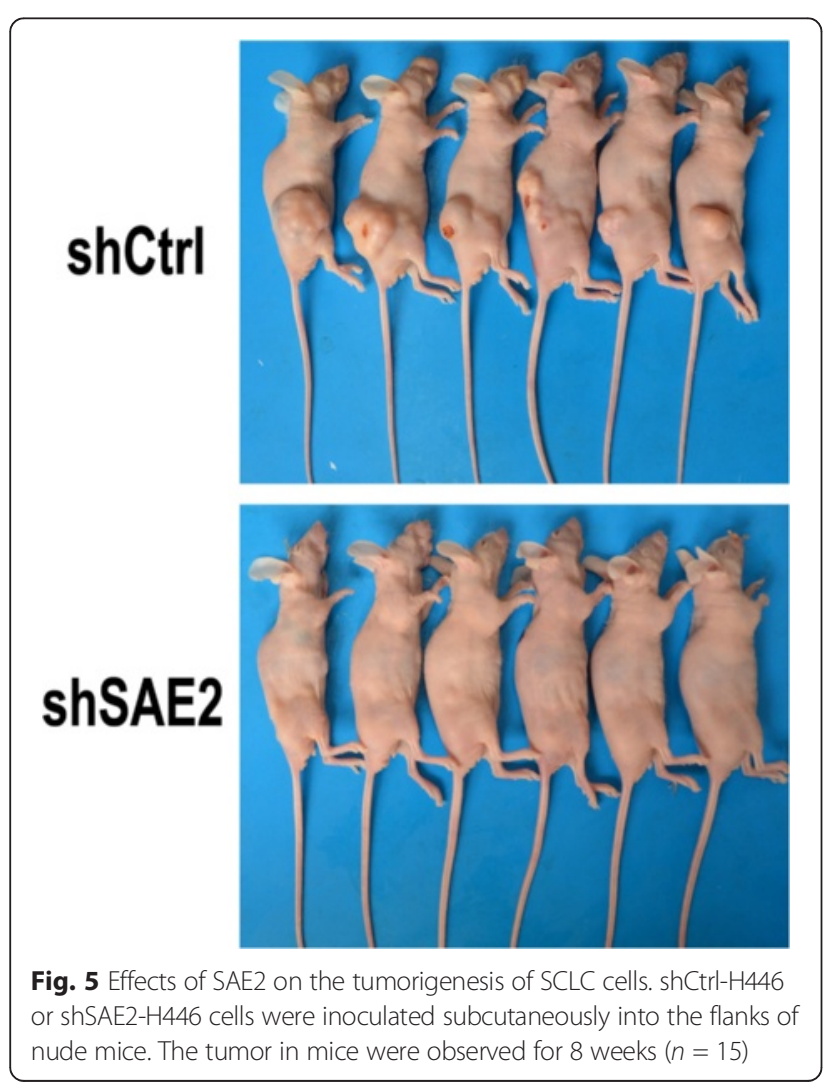

of SAE2 would sensitize SCLC cells to chemotherapy. Cell viability assay for stable cell lines shCtrl-H446 and shSAE2-H446 were performed after treatment with different concentrations of etoposide and cisplatin for 48 and $72 \mathrm{~h}$, respectively (Fig. 6c, d). Our result showed that knockdown of SAE2 in H446 significantly reduced the IC50 of chemotherapeutical agents etoposide (16.65 $\mu \mathrm{M}$ in shSAE2-H446 vs $27.26 \mu \mathrm{M}$ in shCtrl-H446) and cisplatin $(1.874 \mu \mathrm{M}$ in shSAE2-H446 vs $2.528 \mu \mathrm{M}$ in shCtrl-H446) (Table 2). In our previous study, downregulation of SAE2 inhibited cell growth mainly by inducing cell apoptosis, then we examined the apoptosis of SAE2 down-regulated cells treated with etoposide or cisplatin and showed that proportion of apoptotic cells was significantly increased in shSAE2-H446 cells (Fig. 6a, b). These results suggested that down-regulating SAE2 improved chemosensitivity in SCLC.

\section{Discussion}

Although various efforts have been made to improve the treatment of SCLC, the patient prognosis has not been

Table 1 Effects of down-regulation SAE2 expression on subcutaneous tumor-forming rate in nude mice

\begin{tabular}{ll}
\hline Cell line & Incidence \\
\hline shCtrl-H446 & $14 / 15$ \\
ShSAE2-H446 & $0 / 15$ \\
\hline
\end{tabular}




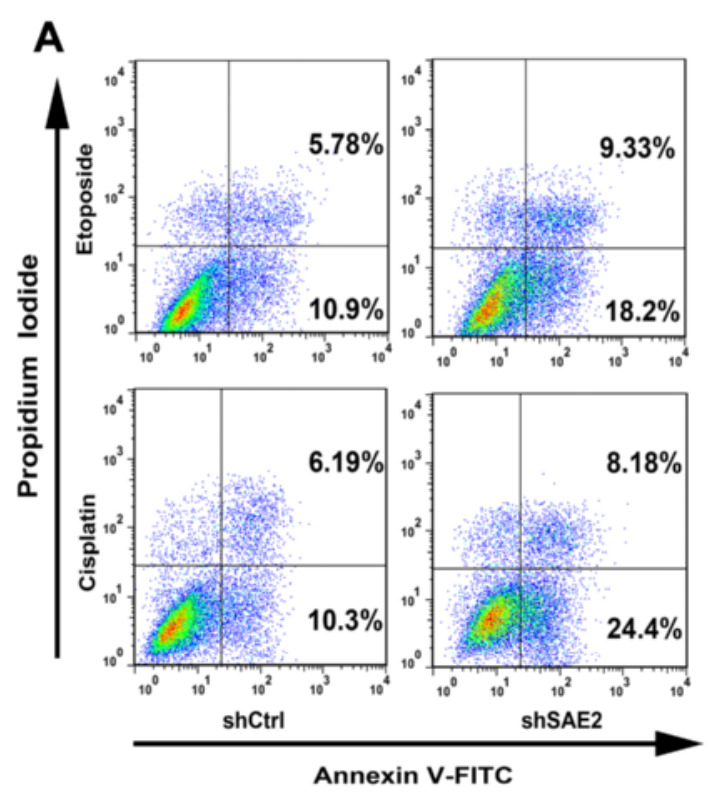

B
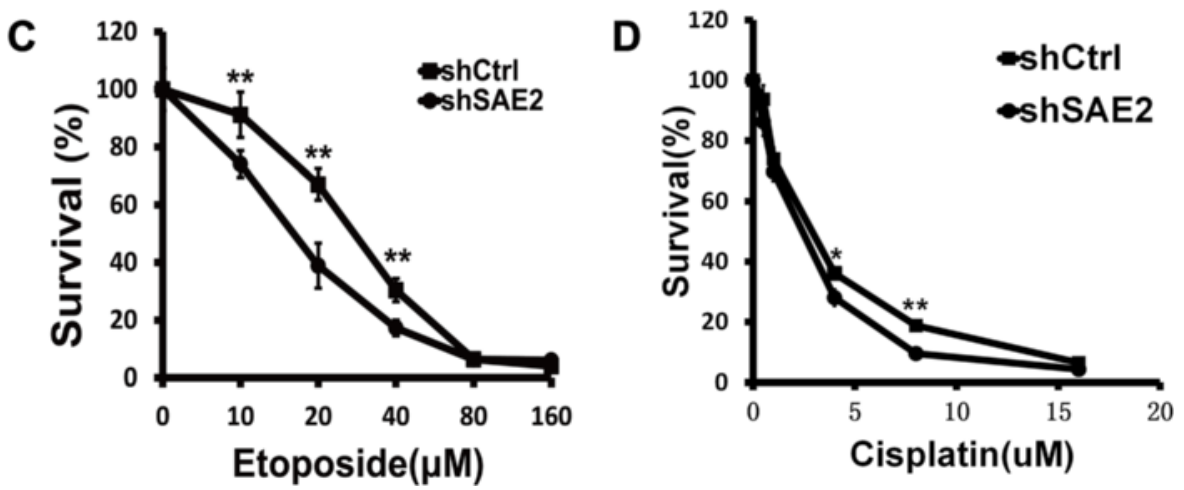

Fig. 6 Knockdown of SAE2 sensitized chemotherapy in H446 (a). shCtrl-H446 and shSAE2-H446 tumor cells were treated with etoposide (15 $\mu$ M) or cisplatin $(1.5 \mu \mathrm{M})$ for 24 and $48 \mathrm{~h}$, respectively. Cell apoptosis was detected by Annexin $\mathrm{V}$ and propidium iodide staining (b). The FCM results are presented as the percentage of apoptotic cells. Drug concentration-dependent cell survival curves for etoposide (c) for $48 \mathrm{~h}$ and cisplatin (d) for $72 \mathrm{~h}$ in shSAE2-H446cells or shCtrl-H446 cells. Each data point represents means \pm SD of three independent experiments ( ${ }^{*} P<0.05$, $\left.{ }^{* *} P<0.01\right)$

improved in the past decades $[1,7,9,10]$. As an essential E1 activating enzyme for SUMOylation, SAE2 plays a key role in SUMOylation which is associated with several diseases including tumors [41]. Recent reports suggest that SAE2 deregulation induces the development of hepatocellular carcinoma [42]. Moreover, Kessler, JD et al. showed that SAE2 inactivation could be a therapeutic strategy in Myc overexpression cancers [31]. Therefore, we speculated that SAE2 was important for tumor formation and progression in SCLC which was characterized with

Table 2 IC50 values of chemotherapeutical agents

\begin{tabular}{lll}
\hline & \multicolumn{2}{c}{ IC50 value $(\mu M)$} \\
\cline { 2 - 3 } Cell line & Cisplatin & Etoposide \\
\hline shCtrl-H446 & 2.528 & 27.26 \\
shSAE2-H446 & 1.874 & 16.65 \\
\hline
\end{tabular}

high c-Myc expression. And we discovered an elevated expression of SAE2 in SCLC tissue and cell lines. We investigated the role of SAE2 in SCLC. Further, we provide the evidence that SAE2 plays an important role in regulating cellular proliferation, invasion, and sensitivity of chemotherapy in SCLC.

Several reports suggested that SUMOylation modification, occurs through a series of enzymatic reactions, is associated with apoptosis regulation, maintenance of genome integrity, modulation of subcellular transport, and transcription [17]. Therefore, the growth inhibition by knocking down SAE2 was assessed, and we discovered that selective down-expression of SAE2 significantly inhibit cell proliferation. Meanwhile, Annexin V-FITC/PI double staining showed an increased cell apoptosis when SAE2 was knocked down. Bcl-2, an important anti-apoptotic protein, was accordingly reduced in shSAE2-H446 stable 
cells, further implying that apoptotic pathway was involved in inhibition of cell proliferation. These data suggest that altered expression of SAE2 is an important contributor to the development of SCLC. Therefore, we analyzed the tumorigenesis of $\mathrm{H} 446$ with or without knockdown of SAE2. Xenograft model showed that selected downexpression of SAE2 significantly decreased tumorigenesis of H446. As demonstrated that misregulation of sumo proteins was involved in tumor development [42], our results suggest that the elevated expression of SAE2 in SCLC cells might contribute to tumorigenesis. This is likely due to the fact that SAE2 is a crucial enzyme for SUMOylation and numerous important proteins, such as tumor oncoproteins.

SCLC has the tendency for early dissemination and metastases [11]. And increasing data have indicated that SUMO modifications were critical regulators in cancerrelated metastasis [23, 30, 41]. In our study, cell migration and invasion assay verified that down-expression of SAE2 inhibited the migratory and invasive properties of SCLC cells in vitro. MMP-2 and MMP-9 are correlated with invasion and metastasis through degradation of type IV collagen which is principal for the basement membrane [43, 44]. Here, we indicated that expression of MMP-2 and MMP-9 was decreased in shSAE2-H446 cells, which suggested that SAE2 silence-induced inhibition of SCLC invasion and migration might be related with MMP-2 and MMP-9. However, the molecular mechanism of shSAE2H446-mediated inhibition of the invasion and metastasis in SCLC needed further research.

Treatment in SCLC is often associated with rapid drug resistance $[5,11]$; therefore, new approaches to improve the efficiency of chemotherapy are extremely needed to be developed. Several studies have suggested that small ubiquitin like modifier SUMOylation is significantly involved in multidrug resistance in several cancers, such as ovarian carcinoma and hepatocellular carcinoma [26, 45, 46]. Consistently, we observed that down-expression of SAE2 significantly sensitized cells to cisplatin and etoposide in vitro. Annexin V-FITC binding assay proved that chemotherapy induced increased cell apoptosis in SAE2 knocked down cells. This may be due to SAE2's role in signal transduction pathways including cytokines, Wnt, NF-кB, and growth factors [18, 42, 47].

Though SAE2 may be a candidate in SCLC treatment, further study needs to be done, especially considering its role dependent on Myc-driven cancers. JD Kessler reported that inactivation of SAE2 inhibited tumorigenicity of Myc-dependent tumors, SUM159 and MDA-MB-231, whereas MCF7 and SKBR3, both of which were Mycindependent, were unaffected. In addition, clinical data showed that expression level of SAE2 correlated with outcome in patient with Myc-high tumors but not Myclow tumors. We also detected the expression of c-Myc in
SCLC cells and found that H446, but not H69 and H526, displayed high expression levels of c-Myc (Additional file 1: Figure S2). Furthermore, silence of SAE2 with siRNA in H526 cells did not induce apoptosis (Additional file 1: Figure S3). This suggests that targeting SAE2 mainly plays a role in SCLC with high c-Myc expression.

\section{Conclusions}

In summary, unprecedentedly, our studies confirmed that SAE2 was aberrantly overexpressed in SCLC significantly correlating to tumorigenesis. Meanwhile, knockdown of SAE2 not only negatively influenced the proliferation, migration, and invasion of SCLC cells but also facilitated basal apoptosis and chemotherapy-induced apoptosis. These findings demonstrate a crucial role of SAE2 in the progression of SCLC and suggest that SAE2 may serve as a clinical biomarker and therapeutic target in SCLC with high c-Myc levels.

\section{Methods}

\section{Cell culture}

Four human SCLC cell lines (H446, H146, H526, and H69) and one normal cell line (BEAS-2B) were used in this study. H446, H146, H526, H69, and BEAS-2B cell lines were cultured in RPMI-1640 Medium containing $10 \%$ fetal bovine serum with $1 \%$ penicillin/streptomycin (SIGMA) at $37{ }^{\circ} \mathrm{C}$ in a $\mathrm{CO}_{2}$ incubator $\left(5 \% \mathrm{CO}_{2}\right)$. Cells in exponential growth phase were used for all experiments.

\section{Immunohistochemical staining}

All the tumor samples of 20 patients with SCLC and 5 normal lung tissues from West China Hospital were fixed in $10 \%$ paraformaldehyde, embedded in paraffin, and cut in $5 \mu \mathrm{m}$ serial sections. Immunohistochemical staining was performed using a peroxidase-labeled streptavidinbiotin technique. Firstly, tissue sections were deparaffinized and rehydrated. Then, sections were boiled in 10 $\mathrm{mM}$ sodium citrate buffer $(\mathrm{pH}$ 6.0) and maintained at a sub-boiling temperature for $10 \mathrm{~min}$ to retrieve antigenicity and were treated with $3 \% \mathrm{H}_{2} \mathrm{O}_{2}$ in methanol for $10 \mathrm{~min}$ to quench endogenous peroxidase activity. After washing in $10 \mathrm{mM}$ PBS (pH 7.6), sections were incubated with $10 \%$ normal mouse serum for SAE2 or rabbit serum (Solarbio Science and Technology) for c-Myc for $10 \mathrm{~min}$ to block nonspecific antibody binding. Sections were then incubated with mouse anti-human SAE2 polyclonal antibody (1:100) or rabbit anti-human c-Myc polyclonal antibody (1:100) overnight at $4{ }^{\circ} \mathrm{C}$. After washing in PBS, sections were treated with a 1:100 dilution of biotinylated donkey antimouse IgG for SAE2 or anti-rabbit IgG for $30 \mathrm{~min}$ followed by a streptavidin-peroxidase conjugate for $30 \mathrm{~min}$. A solution of $0.02 \%$ diaminobenzidine hydrochloride (DAB) containing $0.03 \% \mathrm{H}_{2} \mathrm{O}_{2}$ was used as chromogen to visualize peroxidase activity. The preparations were 
lightly counterstained with hematoxylin, mounted with Permount (Thermo Fisher Scientific), and examined by light microscopy.

\section{Lentiviral vectors mediated SAE2-specific shRNA stable transfection}

Before they were incubated overnight, $293 \mathrm{~T}$ cells were plated at $5 \times 10^{6}$ cells per $100-\mathrm{mm}$ dish. The cells were cotransfected with $9 \mu \mathrm{g}$ pLKO.1-vector/pLKO.1-shSAE2, $9 \mu \mathrm{g}$ psPAX2, and $4.5 \mu \mathrm{g}$ pMD2.G-VSV-G (at a 2:2:1 ratio). Virus was harvested at 48 and 72 h post-transfection, filtered with a $0.45-\mu \mathrm{m}$ pore size cellulose acetate filter (Millipore), and infected the H446 cells with the presence of $8 \mu \mathrm{g} / \mathrm{mL}$ of polybrene. Cells were selected with 0.9 $\mu \mathrm{g} / \mathrm{mL}$ puromycin $24 \mathrm{~h}$ later.

\section{Western blotting}

In brief, cells were washed three times with PBS buffer and lysed in RIPA lysis buffer (Beyotime) on ice. Total protein $(50 \mu \mathrm{g} / \mathrm{sample})$ was extracted and separated by 10 $\%$ sodium dodecyl sulfate-polyacrylamide gel electrophoresis (SDS-PAGE) and then transferred onto polyvinylidene difluoride (PVDF) membranes (Millipore, USA). Membranes were blocked with $5 \%$ non-fat milk in TBST (10 mM Tris, pH $7.4150 \mathrm{mM} \mathrm{NaCl}$, and $0.1 \%$ Tween-20) at room temperature for $1 \mathrm{~h}$. The blotted membranes were incubated with $1-2 \mu \mathrm{g} / \mathrm{ml}$ of primary antibodies (Anti-SAE2:ab118404, Anti-Bcl-2:CST\#2870S,Anti-BclXL:ab32370, Anti-P53:ab32049,Anti-P21:ab109199, Anti MMP2:ab92536, Anti-MMP9:ab3159) diluted in blocking solution at $4{ }^{\circ} \mathrm{C}$ overnight with gentle rocking. After washing five times with TBST, the membrane was reacted with the appropriate HRP-conjugated secondary antibody for $1 \mathrm{~h}$ at $37^{\circ} \mathrm{C}$. $\beta$-actin protein was also determined by using specific antibody (dilution 1:1000, Cell Signaling Technology) as a loading control. After extensive washing with TBST, proteins were visualized by the enhanced chemiluminescence (ECL) detection.

RNA extraction and analysis by quantitative real-time PCR Total RNA from each cell was extracted with RNA simple Total RNA Kit (TIANGEN BIOTECH, BEIJING). The RNA samples were reverse-transcribed into cDNA with the PrimeScript ${ }^{\mathrm{m}} \mathrm{RT}$ reagent Kit (TAKARA). Quantitative real-time PCR was conducted with Bio-rad CFX manager and SsoAdvanced SYBR Green Supermix (Bio-rad) as the detection dye. The primer sequences of PCR were as follows: SAE2, sense 5' -GATAACAGAGCTG CCCGAAAC3' and anti-sense 5' -ATAACACTCGGTCACACCCTTT-3', GAPDH, sense5'GAAGGTGAAGGTCGGAGT-3' and antisense 5' -GAAGATGGTGATGGGATTTC-3'. RT-PCR amplification was performed in 40 cycles with DNA denaturation at $95{ }^{\circ} \mathrm{C}$ for $5 \mathrm{~s}$ and annealing/extension at $60{ }^{\circ} \mathrm{C}$ for $20 \mathrm{~s}$. For analysis, GAPDH mRNA was used to normalize RNA inputs, $\Delta \Delta \mathrm{Ct}$ values were calculated and converted to approximate fold change values (2$\Delta \Delta \mathrm{Ct})$.

\section{Cell proliferation assay}

Seeded in 96-well plates were $2 \times 10^{3}$ cells/well. From the second day to the sixth day, $20 \mu \mathrm{L}$ MTT (Sigma-Aldrich) ( $5 \mathrm{mg} / \mathrm{mL}$ ) was added to each well, incubated at $37{ }^{\circ} \mathrm{C}$ for $4 \mathrm{~h}$, terminated with $150 \mu \mathrm{L}$ of dimethyl sulfoxide (SigmaAldrich) per well, gently shook for $5 \mathrm{~min}$, and determined with an ELISA reader (Bio-Rad) at $570 \mathrm{~nm}$. For cell viable assay, cells were seeded in 96-well plates and exposed to various concentrations of etoposide $(0,5,10,20,40$, 80 , and $160 \mu \mathrm{M})$ for $48 \mathrm{~h}$ or Cisplatin $(0,0.5,1,2,4$, $8,16 \mu \mathrm{M})$ for $72 \mathrm{~h}$; MTT assay was used to detect the chemotherapeutic sensitivity of cells. The concentration for $50 \%$ of maximal effect (IC50 values) was calculated by GraphPad Prism.

\section{Annexin V-binding assay}

Cells were seeded onto 6-well plates at a cell density of $2 \times 10^{5}$ cells/well; 24 h later, cells were treated with Etoposide $(15 \mu \mathrm{M})$ for $24 \mathrm{~h}$ or Cisplatin $(1.5 \mu \mathrm{M})$ for 48 h. Control cells were treated with NS. Then cells were harvested and apoptosis was analyzed by flow cytometry using an FITC Annexin V Apoptosis Detection Kit I (BD Pharmingen) according to the manufacturer's instructions.

\section{Cell cycle analysis}

Cells were seeded at $3 \times 10^{5}$ per well in 6-well plates and cultured with RPMI-1640 Medium non-containing fetal bovine serum for $24 \mathrm{~h}$. Then cells were harvested and washed three times with cold PBS. Cells were fixed in $70 \%$ ice-cold ethanol overnight, washed twice with PBS, stained with PI/RNase staining buffer (BD Pharmingen) at room temperature for $15 \mathrm{~min}$, and detected by flow cytometer. Data were analyzed using CellQuest software.

\section{Cell migration assay}

This cell migration assay was analyzed using transwell cell culture chambers ( $8 \mu \mathrm{m}$ pore size) (Millipore). Briefly, cells $\left(1 \times 10^{5} /\right.$ well $)$ were serum starved for $24 \mathrm{~h}$ and placed in the upper chamber of a 24-well transwell in serum-free medium. RPMI 1640 containing $10 \%$ FBS as chemoattractant were added to the lower chamber, and the cells were incubated at $37^{\circ} \mathrm{C}$ in a $\mathrm{CO}_{2}$ incubator $\left(5 \% \mathrm{CO}_{2}\right.$ ) for $24 \mathrm{~h}$. Then, the filter side of the upper chamber was cleaned with a cotton swab, fixed with $4 \%$ formaldehyde for $10 \mathrm{~min}$, and stained with $0.1 \%$ crystal violet for $20 \mathrm{~min}$. Finally, migrated cells were photographed under a light microscope and counted in six random microscopic fields. 


\section{Cell invasion assay}

Polycarbonate membranes of transwell chambers were coated with Matrigel (BD Biosciences) on the upper surface. Cells $\left(3 \times 10^{4} /\right.$ well $)$ starved from serum for $24 \mathrm{~h}$ were resuspended in serum-free RPMI1640 and added to the upper chamber of 24-well transwell. And a complete RPMI1640 medium was added to the lower chamber as chemoattractant. The cells were incubated for $24 \mathrm{~h}$ at $37{ }^{\circ} \mathrm{C}$ with $5 \% \mathrm{CO}_{2}$. Non-invading cells were removed with a cotton-tipped swab from the top of the Matrigel. The invasive cells were photographed and counted in six random microscopic fields.

\section{RNA interference}

siRNA for SAE2 was designed and synthesized by Life Technologies (Lifetech, China). The sequence of siRNA are as follows: siSAE2-1, sense:5'-GCAGCUGAUGUUCCUCUUAdTdT-3' and anti-sense:3'-dTdTCGUCGACU ACAAGGAGAAU-5'; siSAE2-2, sense:5'-GCUGAGCU CAUAUGGGAUAdTdT-3' and anti-sense: $3^{\prime}$-dTdTCGA CUCGAGUAUACCCUAU-5'. The sequence of negative control (siCtrl) was also designed by Life Technologies. H526 cells were plated onto a 6-well plate at $2 \times 10^{6} /$ well and transfected using GeneSilencer transfection Reagent (Genlantis, CA, USA) according to the manufacturer's protocol. Cells were collected after $48 \mathrm{~h}$ for further experiments.

\section{Tumor formation rate following in vivo transplantation}

A total of $30 \mathrm{Balb} / \mathrm{c}$ nude mice (6 weeks old) were obtained from the Vital River Laboratory Animal Technology, Beijing, housed in a specific pathogen free (SPF) environment; 7 days later, the mice were divided into two groups. The mice were injected with $1 \times 10^{7}$ cells stably knocked down of SAE2 (shSAE2-H446) and parental pLKO.1-vector (shCtrl-H446) cells subcutaneously separately from each group. After inoculation, mice were housed in a sterile barrier system at constant temperature $\left(25 \pm 2{ }^{\circ} \mathrm{C}\right)$ and humidity (45-50 \%). Tumor formation and growth were observed daily.

\section{Colony formation assay}

Cells (single cell suspension) were seeded for colony formation assay in six-well plates at a density of 600 cells/ well. The medium was replaced with fresh medium every 3 days. After 10 days, cell colonies were fixed and stained with crystal violet $(0.5 \%$ in $20 \%$ methanol). Cell colonies were photographed under a light microscope and counted.

\section{Statistical analysis}

Statistical analysis of SAE2 expression level of all 20 patients and 5 controls was performed using SPSS version 19.0 for windows (SPSS Inc) using Mann-Whitney test. All the other statistical analyses were performed using the GraphPad Prism 6.01 software program. Data analysis was carried out using the one-way ANOVA Tukey test for multiple groups and $t$ test for two groups analysis. All data were summarized and presented as means \pm SD or means \pm SEM, $P<0.05$ were considered to indicate statistically significant differences.

\section{Additional file}

Additional file 1: Supplemental materials. Figure S1. Expression levels of SAE2 in SCLC. Figure S2. Expression of c-myc in SCLC cells. Figure S3. Influence of SAE2 on apoptosis in $\mathrm{H} 526$ cells.

\section{Abbreviations}

shRNA: short hairpin RNA; UTR: untranslated region; qRT-PCR: quantitative real-time PCR; MTT: 3-(4,5-dimethylthiazol-2-yl)-2,5-diphenyltetrazolium; SCLC: small cell lung carcinoma; FACS: fluorescence-activated cell sorting; PI: propidium iodide; PBS: phosphate-buffered saline.

\section{Competing interests}

The authors declare that they have no competing interests.

\section{Authors' contributions}

$\mathrm{XKL}$ performed the research and drafted the manuscript and study design with oversight by YSW, YX and ZGP who also carried out the sample selection and immunoassays. QQ, XYL, LJ, PS, and CJF participated in the experiments. YXS participated in the design of the study and performed the statistical analysis. FCG and TY conceived of the study, participated in its design and coordination, and helped to draft the manuscript. All authors read and approved the final manuscript.

\section{Acknowledgements}

The work was supported by the National Natural Science Foundation of China (No.0040205401864)

\section{Author details}

'Department of Thoracic Oncology, Cancer Center, State Key Laboratory of Biotherapy/Collaborative Innovation Center of Biotherapy, West China Hospital, Sichuan University, Chengdu, Sichuan, People's Republic of China. 2Department of Thoracic Oncology, Cancer Center, West China Hospital, Sichuan University, Chengdu, Sichuan, People's Republic of China. ${ }^{3}$ Department of Pathology, West China Hospital, Sichuan University, Chengdu, Sichuan, People's Republic of China.

Received: 19 January 2015 Accepted: 28 May 2015

Published online: 11 June 2015

\section{References}

1. Howlader N, Noone A, Krapcho M, Neyman N, Aminou R, Waldron W, et al. SEER Cancer Statistics Review, 1975-2008. Bethesda: MD: National Cancer Institute; 2011. p. 19.

2. Jemal A, Bray F, Center MM, Ferlay J, Ward E, Forman D. Global cancer statistics. CA Cancer J Clin. 2011;61:69-90.

3. Read WL, Page NC, Tierney RM, Piccirillo JF, Govindan R. The epidemiology of bronchioloalveolar carcinoma over the past two decades: analysis of the SEER database. Lung Cancer. 2004;45:137-42.

4. Devesa SS, Bray F, Vizcaino AP, Parkin DM. International lung cancer trends by histologic type: male: female differences diminishing and adenocarcinoma rates rising. Int J Cancer. 2005;117:294-9.

5. van Meerbeeck JP, Fennell DA, De Ruysscher DK. Small-cell lung cancer. Lancet. 2011;378:1741-55.

6. Albain KS, Crowley J, Livingston R. Long-term survival and toxicity in small cell lung cancer. Expanded Southwest Oncology Group experience. CHEST J. 1991;99:1425-32.

7. Lassen U, Osterlind $K$, Hansen M, Dombernowsky P, Bergman B, Hansen $\mathrm{HH}$. Long-term survival in small-cell lung cancer: posttreatment characteristics in patients surviving 5 to $18+$ years - an analysis of 1,714 consecutive patients. J Clin Oncol. 1995;13:1215-20. 
8. Sher T, Dy GK, Adjei AA. Small cell lung cancer. Mayo Clin Proc. 2008;83:355-67.

9. Jänne PA, Freidlin B, Saxman S, Johnson DH, Livingston RB, Shepherd FA, et al. Twenty-five years of clinical research for patients with limited-stage small cell lung carcinoma in North America. Cancer. 2002;95:1528-38.

10. Chute JP, Chen T, Feigal E, Simon R, Johnson BE. Twenty years of phase III trials for patients with extensive-stage small-cell lung cancer: perceptible progress. J Clin Oncol. 1999;17:1794-801.

11. Jackman DM, Johnson BE. Small-cell lung cancer. Lancet. 2005;366:1385-96.

12. Gustafsson BI, Kidd M, Chan A, Malfertheiner MV, Modlin IM. Bronchopulmonary neuroendocrine tumors. Cancer. 2008;113:5-21.

13. Calbo J, van Montfort E, Proost N, van Drunen E, Beverloo HB, Meuwissen R, et al. A functional role for tumor cell heterogeneity in a mouse model of small cell lung cancer. Cancer Cell. 2011;19:244-56.

14. Gill G. SUMO and ubiquitin in the nucleus: different functions, similar mechanisms? Genes Dev. 2004;18:2046-59.

15. Gareau JR, Lima CD. The SUMO pathway: emerging mechanisms that shape specificity, conjugation and recognition. Nat Rev Mol Cell Biol. 2010;11:861-71.

16. Müller S, Hoege C, Pyrowolakis G, Jentsch S. SUMO, ubiquitin's mysterious cousin. Nat Rev Mol Cell Biol. 2001;2:202-13.

17. Hay RT. SUMO: a history of modification. Mol Cell. 2005;18:1-12.

18. Muller S, Ledl A, Schmidt D. SUMO: a regulator of gene expression and genome integrity. Oncogene. 2004;23:1998-2008.

19. Bossis G, Melchior F. SUMO: regulating the regulator. Cell Div. 2006;1:13.

20. Boggio R, Colombo R, Hay RT, Draetta GF, Chiocca S. A mechanism for inhibiting the SUMO pathway. Mol Cell. 2004;16:549-61.

21. Wang Q, Xia N, Li T, Xu Y, Zou Y, Zuo Y, et al. SUMO-specific protease 1 promotes prostate cancer progression and metastasis. Oncogene. 2012;32:2493-8

22. Mo Y-Y, Yu Y, Theodosiou E, Ee PR, Beck WT. A role for Ubc9 in tumorigenesis. Oncogene. 2005;24:2677-83.

23. Li H, Niu H, Peng Y, Wang J, He P. Ubc9 promotes invasion and metastasis of lung cancer cells. Oncol Rep. 2013;29:1588-94.

24. Bellail AC, Olson JJ, Hao C. SUMO1 modification stabilizes CDK6 protein and drives the cell cycle and glioblastoma progression. Nat Commun. 2014:5:4234.

25. Wiatrek R, Duldulao M, Chen Z, Li W, Nelson R, Kim J, et al. Increased expression of small ubiquitin-like modifier proteins in rectal adenocarcinoma patients after neoadjuvant chemoradiation. J Am Coll Surg. 2011;213:S27.

26. Qin Y, Bao H, Pan Y, Yin M, Liu Y, Wu S, et al. SUMOylation alterations are associated with multidrug resistance in hepatocellular carcinoma. Mol Med Reports. 2014;9:877-81.

27. Cheng J, Bawa T, Lee P, Gong L, Yeh ET. Role of desumoylation in the development of prostate cancer. Neoplasia. 2006;8:667-76.

28. Bogachek MV, Chen Y, Kulak MV, Woodfield GW, Cyr AR, Park JM, et al. Sumoylation pathway is required to maintain the basal breast cancer subtype. Cancer cell. 2014;25:748-61.

29. Bawa-Khalfe T, Yeh ET. SUMO losing balance: SUMO proteases disrupt SUMO homeostasis to facilitate cancer development and progression. Genes Cancer. 2010;1:748-52.

30. Baek SH. A novel link between SUMO modification and cancer metastasis. Cell Cycle. 2006;5:1492-5.

31. Kessler JD, Kahle KT, Sun T, Meerbrey KL, Schlabach MR, Schmitt EM, et al. A SUMOylation-dependent transcriptional subprogram is required for Myc-driven tumorigenesis. Science. 2012;335:348-53.

32. Rudin CM, Poirier JT. MYC, MAX, and small cell lung cancer. Cancer Discovery. 2014;4:273-4.

33. Saksela K, Bergh J, Lehto V-P, Nilsson K, Alitalo K. Amplification of the c-myc oncogene in a subpopulation of human small cell lung cancer. Cancer Res. 1985:45:1823-7.

34. Prins J, De Vries E, Mulder N. The myc family of oncogenes and their presence and importance in small-cell lung carcinoma and other tumour types. Anticancer Res. 1992;13:1373-85.

35. Little CD, Nau MM, Carney DN, Gazdar AF, Minna JD. Amplification and expression of the c-myc oncogene in human lung cancer cell lines. Nature. 1983;306:194-6.

36. Johnson BE, Russell E, Simmons AM, Phelps R, Steinberg SM, Ihde DC, et al. MYC family DNA amplification in 126 tumor cell lines from patients with small cell lung cancer. J Cell Biochem. 1996;63:210-7.
37. Johnson BE, Makuch RW, Simmons AD, Gazdar AF, Burch D, Cashell AW. Myc family DNA amplification in small cell lung cancer patients' tumors and corresponding cell lines. Cancer Res. 1988;48:5163-6.

38. Brennan J, O'Connor T, Makuch RW, Simmons AM, Russell E, Linnoila RI, et al. Myc family DNA amplification in 107 tumors and tumor cell lines from patients with small cell lung cancer treated with different combination chemotherapy regimens. Cancer Res. 1991;51:1708-12.

39. Barr LF, Campbell SE, Bochner BS, Dang CV. Association of the decreased expression of alpha3beta1 integrin with the altered cell: environmental interactions and enhanced soft agar cloning ability of c-myc-overexpressing small cell lung cancer cells. Cancer Res. 1998;58:5537-45.

40. de Cassia SAR, Meurer RT, Roehe AV. MYC amplification is associated with poor survival in small cell lung cancer: a chromogenic in situ hybridization study. J Cancer Res Clin Oncol. 2014;140:2021-5.

41. Kim JH, Choi HJ, Kim B, Kim MH, Lee JM, Kim IS, et al. Roles of sumoylation of a reptin chromatin-remodelling complex in cancer metastasis. Nat Cell Biol. 2006:8:631-9.

42. Hoeller D, Hecker C-M, Dikic I. Ubiquitin and ubiquitin-like proteins in cancer pathogenesis. Nat Rev Cancer. 2006;6:776-88.

43. Itoh $Y$, Nagase H. Matrix metalloproteinases in cancer. Essays Biochem. 2002:38:21-36.

44. Curran S, Murray GI. Matrix metalloproteinases: molecular aspects of their roles in tumour invasion and metastasis. Eur J Cancer. 2000;36:1621-30.

45. Huang RY, Kowalski D, Minderman H, Gandhi N, Johnson ES. Small ubiquitin-related modifier pathway is a major determinant of doxorubicin cytotoxicity in Saccharomyces cerevisiae. Cancer Res. 2007;67:765-72.

46. Helleman J, Jansen MP, Span PN, van Staveren IL, Massuger LF, Meijer-van Gelder ME, et al. Molecular profiling of platinum resistant ovarian cancer. Int J Cancer. 2006;118:1963-71.

47. Stehmeier $\mathrm{P}$, Muller $\mathrm{S}$. Regulation of p53 family members by the ubiquitinlike SUMO system. DNA Repair. 2009;8:491-8.

\section{Submit your next manuscript to BioMed Central and take full advantage of:}

- Convenient online submission

- Thorough peer review

- No space constraints or color figure charges

- Immediate publication on acceptance

- Inclusion in PubMed, CAS, Scopus and Google Scholar

- Research which is freely available for redistribution 